\title{
Editorial
}

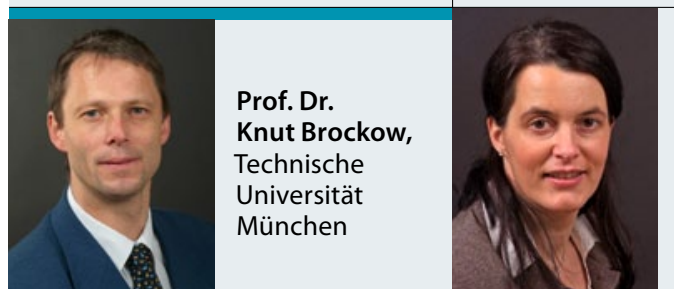

Prof. Dr. Claudia TraidlHoffmann,

Technische Universität München

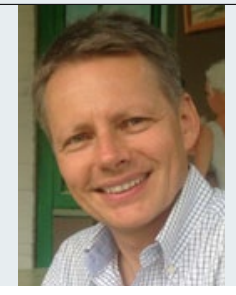

Prof. Dr. Markus Ollert, Technische Universität München
Prof. Dr. Thilo Jakob, Universitätsklinikum Freiburg

\section{Ehre, wem Ehre gebührt!}

Z um Jahreswechsel wird Prof. Dr. med. Dr. phil. Johannes Ring vom aktiven Dienst als Direktor der Klinik und Poliklinik für Dermatologie und Allergologie am Biederstein zurücktreten, nachdem er etwa 35 Jahre die Allergologie und Dermatologie in Deutschland und weit darüber hinaus geprägt hat. Durch sein herausragendes Engagement in der klinischen und in der Grundlagenforschung sowie durch seine unermüdliche Gremienund Öffentlichkeitsarbeit hat er maßgeblich dazu beigetragen, die Allergologie zu dem zu machen, was sie heute ist. Hier hat er u. a. zehn Jahre als Präsident die Geschicke der Deutschen Gesellschaft für Allergologie und klinische Immunologie (DGAKI) geleitet, war Vizepräsident der World Allergy Organization (WAO), Präsident des elitären Collegium Internationale Allergologicum (CIA), der European Academy of Dermatology and Venereology (EADV) und der European Society for Dermatological Research (ESDR). Seine wissenschaftliche Laufbahn hat ihn von den Anfängen in der experimentellen Immunologie über die Erforschung von Arzneimittelnebenwirkungen und Anaphylaxie zur Epidemiologie allergischer Erkrankungen, zum atopischen Ekzem mit Entwicklung des Atopie-Patch-Tests bis hin zur Entwicklung der Neurodermitis- und Anaphylaxieschulung geführt. Hierbei zeigte er immer ein bewundernswertes Zusammenspiel an Motivation, Einsatz und Disziplin, aber auch stets eine große Offenheit und Interesse gegenüber neuen Ideen und Entwicklungen. Er ist als Lehrer überzeugend, motivierend und mitreißend, erlaubt jedoch auch viele Freiheiten für eigene Ideen und Projekte - Tugenden, die nur wenige wirklich zu leben verstehen. Ein besonderes Anliegen war nicht nur die translationale Forschung sondern auch die translationale Fortbildung zu allergologischen Themen, in denen Expertenwissen für den klinisch tätigen Allergologen übersetzt wurden. Zusammen mit Gerhard Schultze-Werninghaus gründete er hierfür 1991 das Allergo Journal als Wissenschaftsorgan der DGAKI und des AeDA (Ärzteverband Deutscher Allergologen) und machte in zehnjährigen Herausgeberschaft das Allergo Journal zu der am meisten gelesenen wissenschaftlichen Fachzeitschrift auf dem Gebiet der Allergologie, die mit einer Auflage von 8.000 Exemplaren die meisten allergologisch tätigen und interessierten Ärzte in Deutschland erreicht.

Seine Mitarbeiter und Schüler organisieren ihm zu Ehren am 9. Dezember 2013 in München das erste Internationale Ring Symposium „Skin in Inflammation and Allergy", bei dem die neuesten Resultate der allergologischen, immunologischen und dermatologischen Forschung präsentiert werden. Dass Begeisterung für Allergologie und Dermatologie ansteckend ist und sich Johannes Ring nicht nur den Respekt, sondern auch die Freundschaft von den hochrangigsten Forschern international verdient hat, zeigt sich u. a. an der langen Liste der Vertreter aus der "Champions League“ der Allergologie, Immunologie und Dermatologie, die ihre aktive Teilnahme zugesagt haben. Als Zeichen der Verbundenheit haben die meisten Johannes Ring zu Ehren eine kleine Festschrift verfasst, die wir Ihnen in dieser Ausgabe präsentieren können. Hier geht es von hämatopoetischen Vorläuferzellen der eosinophilen und basophilen Granulozyten als Risikomarker für Atopie über die Mikrobiom-gastrointestinale-zentralnervöse Achse zur personalisierten Medizin des atopischen Ekzems und der verzögerten Anaphylaxie auf tierische Nahrungsmittel bis hin zu neuen Entwicklungen in der Therapie maligner Hauterkrankungen.

Zusätzlich ist in der aktuellen Ausgabe die erste Deutschlandkarte der Vergütungen für allergologische Leistungen (S. 588) veröffentlicht - eine höchst spannende Zusammenstellung, die einem den Irrsinn des Föderalismus im Bereich der Kassenärztlichen Vereinigungen vor Augen führt. Ab Seite 546 finden Sie den zwölften Teil der Serie „Molekulare Allergologie", diesmal zu dem wichtigen Thema Hausstaubmilbenallergene. Ab Seite 570 können Sie sich zur Bedeutung der In-vitro-Diagnostik bei der Beurteilung berufsbedingter allergischer Erkrankungen fortbilden. Wir wünschen unseren Leserinnen und Lesern viel Freude bei der Lektüre.
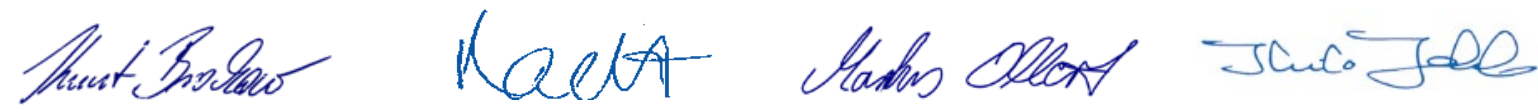